\title{
Piezo-optic tensor of crystals from quantum-mechanical calculations
}

\author{
A. Erba, ${ }^{1, a)}$ M. T. Ruggiero, ${ }^{2}$ T. M. Korter, ${ }^{2}$ and R. Dovesi ${ }^{1}$ \\ ${ }^{1}$ Dipartimento di Chimica, Università di Torino and NIS, Nanostructured Interfaces and Surfaces, \\ Centre of Excellence, Via Giuria 5, 10125 Torino, Italy \\ ${ }^{2}$ Department of Chemistry, Syracuse University, 1-014 Center for Science and Technology, \\ Syracuse, New York 13244-4100, USA
}

(Received 3 September 2015; accepted 29 September 2015; published online 12 October 2015)

\begin{abstract}
An automated computational strategy is devised for the ab initio determination of the full fourth-rank piezo-optic tensor of crystals belonging to any space group of symmetry. Elastic stiffness and compliance constants are obtained as numerical first derivatives of analytical energy gradients with respect to the strain and photo-elastic constants as numerical derivatives of analytical dielectric tensor components, which are in turn computed through a Coupled-Perturbed-Hartree-Fock/Kohn-Sham approach, with respect to the strain. Both point and translation symmetries are exploited at all steps of the calculation, within the framework of periodic boundary conditions. The scheme is applied to the determination of the full set of ten symmetry-independent piezo-optic constants of calcium tungstate $\mathrm{CaWO}_{4}$, which have recently been experimentally reconstructed. Present calculations unambiguously determine the absolute sign (positive) of the $\pi_{61}$ constant, confirm the reliability of 6 out of 10 experimentally determined constants and provide new, more accurate values for the remaining 4 constants. (C) 2015 AIP Publishing LLC. [http://dx.doi.org/10.1063/1.4932973]
\end{abstract}

\section{INTRODUCTION}

Several optical properties of anisotropic media are related to birefringence or double refraction. It is indeed well-known that two plane polarized electro-magnetic waves, with two distinct velocities $v$, may in general be propagated through a crystal with the same propagation direction. The $c / v$ ratio for each wave ( $c$ being the speed of light) is referred to as the refractive index for that wave and can be derived for any propagation direction from the so-called optical indicatrix: an ellipsoid oriented along the principal axes of the dielectric tensor $\boldsymbol{\epsilon}$ and whose semi-axes give the principal refractive indices of the system. ${ }^{1}$ It follows that any change in the shape, size, and orientation of the indicatrix results in the modification/modulation of the optical properties of a crystal. Such changes can be induced by electric fields, strains and stresses giving rise to the electro-optic, elasto-optic (i.e., photo-elastic), and piezo-optic effects, respectively.

In particular, the piezo-optic effect (measuring the variation of the dielectric tensor components as induced by an applied stress) has proven to be an effective tool for mapping $2 \mathrm{D}$ and $3 \mathrm{D}$ mechanical stresses through stress tensorfield tomography. ${ }^{2,3}$ Furthermore, such an effect is extremely relevant to the field of optoelectronics where the search for highly efficient electro-optic and piezo-optic materials is experiencing a great interest in recent years due to their applications as photo-elastic modulators of light polarization and as components of many devices related to acousto-optic light modulators, deflectors, tunable spectral filters, etc. ${ }^{4-11}$ The piezo-optic and photo-elastic responses of a crystal are quite anisotropic even for high-symmetry systems. In order to accurately optimize the orientation of a crystal, which maximizes

\footnotetext{
a)Electronic mail: alessandro.erba@unito.it
}

the efficiency of the acousto-optic transformation, absolute values, and signs of all non-zero elements of the fourth-rank elastic $\mathbb{C}$, compliance $\mathbb{S}$, photo-elastic $\mathbf{p}$, and piezo-optic $\pi$ tensors have to be determined, which is not a trivial task (see below), especially for low-symmetry crystals. ${ }^{10,12,13}$

From an experimental point of view, photo-elastic constants could be measured through acousto-optic methods. Within the phenomenological theory of Brillouin scattering, for instance, photo-elastic constants are given in terms of the intensities of Brillouin components. ${ }^{14,15}$ Acousto-optic methods, however, are unable to uniquely determine all signs of the photo-elastic constants, particularly so for low-symmetry crystals. ${ }^{4,12}$ The full set of independent piezo-optic constants can be measured for crystals of any symmetry via the combination of optical interferometry and polarization-optical techniques (once the elastic compliances are known). ${ }^{12,16-18}$ Still, this approach requires a large number of measurements to be performed on a large number of crystalline samples (properly cut to specific orientations). In their fundamental work of 2005, Andrushchak et al. ${ }^{12}$ derived the minimum set of samples and measurements necessary to determine the whole piezo-optic tensor for all crystal families: 57 measurements on 16 samples are required to determine the 36 independent components of a triclinic crystal, 29 measurements on 8 samples for the 20 independent components of a monoclinic crystal, 14 measurements on 6 samples for the 10 independent components of tetragonal crystals belonging to the $4, \overline{4}$ or $4 / m$ classes, just to name a few. Even small errors in the sample cut preparation or in the alignment of the whole optical setup may result in large errors in the piezo-optic constants determination (see the detailed analysis on the possible sources of inaccuracies given by Krupych et al. in Ref. 16). Furthermore, the interferometric method is known to provide a somewhat poor description of so-called rotating piezo-optical constants 
(those which correspond to a rotation of the optical indicatrix under mechanical stress); a conoscopic method has recently been devised to determine such constants more accurately. ${ }^{19}$

In this paper we present a general, fully automated scheme for the theoretical $a b$ initio calculation of the fourth-rank piezo-optic tensor $\pi$ of crystals belonging to any space group symmetry, which requires the simultaneous determination of the elastic stiffness and compliance tensors and of the photoelastic tensor. The scheme adopts periodic-boundary conditions, fully exploits both point and translational symmetries at all steps, ${ }^{20-23}$ is based on the evaluation of analytical energy gradients with respect to both atomic coordinates and lattice parameters ${ }^{24-27}$ and utilizes the analytical CoupledPerturbed-Hartree-Fock/Kohn-Sham (CPHF/KS) method to compute static and electric field frequency-dependent optical (i.e., dielectric) properties of crystals. ${ }^{28-30}$ We have implemented the present scheme into a development version of the public CRYSTAL14 program, ${ }^{31,32}$ taking advantage of the many recent developments made to the algorithms for the evaluation of strain-related tensorial properties (elastic, piezoelectric, photo-elastic) of crystals. ${ }^{33-38}$

The proposed scheme is first described in detail and then applied to the determination of the piezo-optic tensor of tetragonal calcium tungstate, $\mathrm{CaWO}_{4}$, which belongs to the scheelite family of minerals. This is a promising material for acoustooptic modulators and its full set of piezo-optic (and photoelastic) constants has been experimentally determined only quite recently. ${ }^{13,17-19}$ An explicit investigation is performed on the effect of adopting several functionals of density functional theory (DFT) belonging to three rungs of the so-called "Jacob's ladder"39 (namely, a local-density approximation, a generalized-gradient approximation, and a couple of global hybrids), and on the electric field frequency-dependence of all computed dielectric, piezo-optic, and photo-elastic properties. Our calculations allow us to confirm the experimentally measured values of a sub-set of the 10 independent piezo-optic and photo-elastic constants of $\mathrm{CaWO}_{4}$ and, on the contrary, to point-out large discrepancies in the determination of the others and to provide a complete description of the anisotropic stressoptical response of this crystal.

\section{THEORY AND METHODS}

\section{A. Stress-related tensorial properties of crystals and their symmetry features}

The stress-strain relation for an anisotropic crystal is given by the generalized Hooke's law,

$$
\sigma_{i j}=C_{i j k l} \eta_{k l}
$$

which relates the second-rank stress tensor $\sigma$ to the secondrank pure strain tensor $\boldsymbol{\eta}$ through the 81 elastic stiffness constants $C_{i j k l}$ that constitute the fourth-rank elastic tensor $\mathbb{C}$. In the above expression, $i, j, k, l$ are Cartesian indices. As both $\sigma$ and $\boldsymbol{\eta}$ are symmetric tensors, the number of their independent components reduces from 9 to 6 , and those of $\mathbb{C}$ accordingly from 81 to 36. As a consequence, Voigt's notation can be used, which introduces a mapping between pairs of Cartesian indices and Voigt's indices running from 1 to $6: v, u=1, \ldots, 6$ where

$$
\begin{gathered}
1=x x, 2=y y, 3=z z, 4=y z, 5=x z \text { and } 6=x y:^{1} \\
\sigma_{v}=C_{v u} \eta_{u} \quad \text { and } \quad \eta_{v}=S_{v u} \sigma_{u},
\end{gathered}
$$

where the fourth-rank elastic tensor has been given a $6 \times 6$ matrix representation and where $\mathbb{S}=\mathbb{C}^{-1}$ represents the socalled elastic compliance tensor. To second-order in the strain, the elastic constants can be defined as second energy density derivatives with respect to pairs of strains,

$$
C_{v u}=\frac{1}{V}\left(\frac{\partial^{2} E}{\partial \eta_{v} \partial \eta_{u}}\right),
$$

being $V$ the volume of the crystal cell. From Equation (3), the equivalence $C_{v u} \equiv C_{u v}$ is evident, which makes the $6 \times 6 \mathbb{C}$ matrix symmetric and reduces the number of its independent components to 21. Neumann's principle, which states that any physical property of a crystal must exhibit at least the same point-symmetry of the crystal, can be exploited to further reduce the number of independent components by imposing the invariance $\left(\mathbb{C}^{R}=\mathbb{C}\right)$ of the fourth-rank elastic tensor to the action of any point-symmetry operator $\hat{R}$ of the point-group to which the crystal belongs,

$$
\mathbb{C}_{i j k l}^{R}=r_{i p} r_{j q} r_{k s} r_{l t} \mathbb{C}_{p q s t},
$$

where $r$ is the $3 \times 3$ Cartesian matrix representation of the symmetry operator $\hat{R}$ and Einstein's notation is used according to which repeated indices are meant to be summed. By imposing the invariance of $\mathbb{C}$ with respect to all the symmetry operators of the $4 / m$ class of the tetragonal crystal system to which $\mathrm{CaWO}_{4}$ belongs, the following shape of the elastic tensor is obtained, for instance,

$$
\mathbb{C}=\left(\begin{array}{cccccc}
C_{11} & C_{12} & C_{13} & 0 & 0 & C_{16} \\
C_{12} & C_{11} & C_{13} & 0 & 0 & -C_{16} \\
C_{13} & C_{13} & C_{33} & 0 & 0 & 0 \\
0 & 0 & 0 & C_{44} & 0 & 0 \\
0 & 0 & 0 & 0 & C_{44} & 0 \\
C_{16} & -C_{16} & 0 & 0 & 0 & C_{66}
\end{array}\right) .
$$

In the present implementation, elastic constants in Equation (3) are computed as numerical first-derivatives of analytical energy gradients. ${ }^{33,40}$

The variation of the components of the inverse dielectric tensor $\boldsymbol{\epsilon}^{-1}$ as induced by strain is given by the strain-optical coefficients (i.e., elements of the fourth-rank Pockels' elastooptic or photo-elastic tensor $\mathbf{p}$ ),

$$
\Delta \epsilon_{i j}^{-1}=p_{i j k l} \eta_{k l} \text {. }
$$

As both the inverse dielectric tensor $\boldsymbol{\epsilon}^{-1}$ and the pure strain tensor $\boldsymbol{\eta}$ are symmetric, Voigt's notation can be adopted also in this case so that the photo-elastic tensor $\mathbf{p}$ can be given a $6 \times 6$ matrix representation as for the elastic tensor. Photo-elastic constants can then be derived according to

$$
p_{v u}=\frac{\partial \epsilon_{v}^{-1}}{\partial \eta_{u}},
$$

where, in the present scheme, the derivative with respect to the strain is evaluated numerically while the dielectric tensor ( $\boldsymbol{\epsilon}$ $=1+4 \pi \alpha / V)$ analytically from the polarizability $\alpha$ determined through the $\mathrm{CPHF} / \mathrm{KS}$ method (for a closed-shell 
system), ${ }^{31}$

$$
\alpha_{i j}=-\frac{2}{n_{k}} \sum_{\mathbf{k}}^{\mathrm{BZ}} \mathfrak{R}\left\{\operatorname{Tr}\left(C^{\mathbf{k} \dagger} \Omega^{\mathbf{k}, i} C^{\mathbf{k}} \bigcup^{\mathbf{k}, j} n\right)\right\},
$$

where $n_{k}$ is the number of $\mathbf{k}$ points in the first Brillouin Zone (BZ) and $n$ is the diagonal occupation matrix whose elements are 2 for occupied orbitals and 0 otherwise. Here $U^{\mathbf{k}, j}$ is an anti-Hermitian block off-diagonal matrix that relates the unperturbed coefficient matrix $C^{\mathbf{k}}$, to the corresponding first-order perturbed matrix, $C^{\mathbf{k}, j} \equiv C^{\mathbf{k}} \mathbf{U}^{\mathbf{k}, j}$, which gives the linear (firstorder) response to the electric field perturbation represented by the matrix $\Omega^{\mathbf{k}, i}$. The off-diagonal blocks of $\mathrm{U}^{\mathbf{k}, j}$ depend not only on $\Omega^{\mathbf{k}, j}$ but also on the first-order perturbed density matrix (through the two-electron terms in the Hamiltonian) which, in turn, depends upon $\mathrm{U}^{\mathbf{k}, j}$. Hence, a self-consistent solution of the $\mathrm{CPHF} / \mathrm{KS}$ equations is required.

As recalled in the introduction, the dielectric tensor $\boldsymbol{\epsilon}$ determines the optical indicatrix of the crystal. For cubic crystals the indicatrix is a sphere. For hexagonal, tetragonal (as $\mathrm{CaWO}_{4}$ ), and trigonal crystals (optically uniaxial), the indicatrix is an ellipsoid of revolution about the principal symmetry axis of the system (also called optic axis). The section of the indicatrix perpendicular to the optic axis is a circle whose radius $n_{o}$ is the ordinary refractive index of the system while the section perpendicular to any other direction is an ellipse whose semi-axes are $n_{o}$ and $n_{e}$, the latter being the extraordinary refractive index. Calcium tungstate is said to be an optically positive crystal as $n_{e}>n_{o}$. The birefringence of a material can be defined as $\delta=n_{e}-n_{o}$.

Given stress-strain relation (2), the fourth-rank piezooptic tensor $\pi$ (whose elements are the stress-optical coefficients $\pi_{v u}$ ) can be obtained from the photo-elastic $\mathbf{p}$ and elastic $\mathbb{C}$ ones as ${ }^{1}$

$$
\boldsymbol{\pi}=\mathbf{p} \mathbb{S} \quad \text { and conversely } \quad \mathbf{p}=\boldsymbol{\pi} \mathbb{C} .
$$

At variance with the elastic $\mathbb{C}$ and compliance $\mathbb{S}$ tensors, $\mathbf{p}$ and $\pi$ are not symmetric (i.e., in general $p_{v u} \neq p_{u v}$ and $\pi_{v u} \neq \pi_{u v}$ ). It follows that the number of symmetry-independent components to be determined for the stress-optical and strain-optical tensors is generally larger than for the elastic tensors. The invariance of the piezo-optic tensor with respect to all the point-symmetry operators $\hat{R}$ of the crystal has to be imposed $\left(\boldsymbol{\pi}^{R}=\boldsymbol{\pi}\right)$ without the constraint of being symmetric,

$$
\pi_{i j k l}^{R}=r_{i p} r_{j q} r_{k s} r_{l t} \pi_{p q s t}
$$

For instance, a triclinic crystal has 36 independent piezo-optic coefficients, with the number of unique values decreasing with increasing symmetry. In the case of $\mathrm{CaWO}_{4}$ (tetragonal $4 / m$ ), the shape of the piezo-optic tensor is

$$
\boldsymbol{\pi}=\left(\begin{array}{ccc|ccc}
\pi_{11} & \pi_{12} & \pi_{13} & 0 & 0 & \pi_{16} \\
\pi_{12} & \pi_{11} & \pi_{13} & 0 & 0 & -\pi_{16} \\
\pi_{31} & \pi_{31} & \pi_{33} & 0 & 0 & 0 \\
\hline 0 & 0 & 0 & \pi_{44} & \pi_{45} & 0 \\
0 & 0 & 0 & -\pi_{45} & \pi_{44} & 0 \\
\pi_{61} & -\pi_{61} & 0 & 0 & 0 & \pi_{66}
\end{array}\right) .
$$

The piezo-optic coefficients $\pi_{v u}$ where both $v, u=1,2,3$ describe the connection between the principal refractive indices and the normal stresses and are referred to as principal coefficients. Coefficients $\pi_{v u}$ where $v=1,2,3$ and $u=4,5,6$ relate the principal refractive indices to shear stresses and are called shifting coefficients. Piezo-optic coefficients where $v$ $=4,5,6$ correspond to the rotation of the optical indicatrix and are called rotating or rotating-shifting coefficients depending on whether $u=1,2,3$ or $u=4,5,6$, respectively. ${ }^{3}$ Photo-elastic constants $p_{v u}$ are dimensionless while piezo-optic $\pi_{v u}$ ones are generally expressed in units of Brewsters, where $1 \mathrm{Br}$ $=10^{-12} \mathrm{~Pa}^{-1}=1 \mathrm{TPa}^{-1}$.

\section{B. The automated algorithm}

One of the specific features of the present scheme for the evaluation of the piezo-optic fourth-rank tensor of crystals of any symmetry is its fully automated character, which requires a "one-shot" calculation of elastic, dielectric and photo-elastic constants, and their combination. Such a scheme involves the efficient integration of several fundamental algorithms, such as analytic energy gradients, geometry optimizations for the relaxation of atomic positions within strained and unstrained configurations, and $\mathrm{CPHF} / \mathrm{KS}$ self-consistentfield procedures. As for elastic, piezoelectric, and photoelastic constants, piezo-optic ones can also be decomposed into purely electronic "clamped-ion" and nuclear "internalstrain" contributions where the latter measures the piezo-optic effect due to the nuclear relaxation induced by strain, ${ }^{41,42}$ which is accounted for by optimizing the atomic positions within the strained cell. Experimental measurements of the static dielectric and piezo-optic response properties of crystals should refer to the limit of infinite electric-field wavelength $\lambda$. Practically, they are performed at finite values of $\lambda$, which are expected to correspond to sufficiently high frequencies of the applied electric field to make nuclear contributions negligible, but not high enough for promoting electronic excitations. These two constraints are such that the adopted values of $\lambda$ often do not correspond to the $\lambda \rightarrow \infty$ limit of the static CPHF/KS calculations. In the present implementation, we take advantage of recent developments on the evaluation of "dynamic" dielectric properties of solids ${ }^{31,43,44}$ to explicitly investigate the effect of $\lambda$ on computed piezo-optic constants.

The resulting algorithm can be sketched as follows: (i) the starting crystal structure is fully optimized at the selected level of theory; (ii) cell gradients are computed on the optimized unstrained configuration as well as the equilibrium dielectric tensor (which is then inverted) with the $\mathrm{CPHF} / \mathrm{KS}$ scheme (by default $\lambda=\infty$ ); (iii) a full symmetry analysis is performed in order to determine the non-null independent elastic and piezo-optic constants. The minimal set of independent deformations to be applied, out of a maximum of six, is also found; (iv) a given deformation is selected and the corresponding residual symmetry determined; for each deformation, $N_{s}$ strained configurations are defined according to a strain amplitude $a$ (by default, $N_{s}=2$, corresponding to one "expanded" and one "contracted" configuration with a strain amplitude $a=0.015$, i.e., a deformation of $1.5 \%$ 
with respect to the unstrained lattice); (v) for each strained configuration, atomic positions are relaxed (default option) or not depending on whether one wants to go beyond or not the "clamped-ion" approximation; cell gradients and dielectric tensor of the strained configurations are computed; (vi) cell gradients and inverse dielectric tensors along a given deformation are fitted with singular-value-decomposition routines, their derivatives determined numerically and the corresponding elastic and photo-elastic constants obtained; (vii) once all independent deformations have been considered and all independent elastic and photo-elastic constants determined, full elastic and photo-elastic tensors are built based on the symmetry analysis performed at the beginning, the elastic tensor is inverted and the piezo-optic constants obtained by combining the photo-elastic with the compliance tensors.

\section{Computational setup}

The algorithm described in Section II B has been implemented into a development version of the CRYSTAL14 program, ${ }^{31}$ which adopts a basis set of localized Gaussiantype functions (GTF). An all-electron basis set has been adopted for describing $\mathrm{O}$ and $\mathrm{Ca}$ atoms, which consists in a $6-31 \mathrm{G}(2 d)$ contraction and a $6-31 \mathrm{G}(d)$ contraction of GTFs, respectively. The large-core effective pseudo-potential derived by Hay and Wadt has been chosen for tungsten, which leaves only the $5 d$ and $6 s p$ valence electrons to be explicitly described. A split-valence basis set has been adopted, composed of two independent, single Gaussian $s p$ shells, and a $3-1 \mathrm{G}$ contraction for $d$ electrons. ${ }^{45}$ Four different formulations of exchange-correlation DFT functional are considered: the local-density approximation (LDA), SVWN functional, ${ }^{46,47}$ the generalized-gradient approximation (GGA), $\mathrm{PBE}^{48}$ functional, and hybrid B3LYP ${ }^{49}$ and $\mathrm{PBE}^{50}$ functionals. Thresholds controlling the accuracy of Coulomb and exchange series are set to $8,8,8,8,16 .{ }^{32}$ Reciprocal space is sampled using a Monkhorst-Pack mesh with a shrinking factor of 6 for the primitive cell of $\mathrm{CaWO}_{4}$, corresponding to 36 independent k-points in the irreducible portion of the Brillouin zone. A pruned grid with 1454 radial and 99 angular points is used to calculate the DFT exchange-correlation contribution through numerical integration of the electron density over the unit cell volume. ${ }^{32}$ The self-consistent field convergence on energy was set to a value of $10^{-8}$ hartree for all geometry optimizations.

\section{RESULTS AND DISCUSSION}

Before discussing stress- or strain-induced anisotropic responses (elasticity, photo-elasticity, piezo-optics) of calcium tungstate, $\mathrm{CaWO}_{4}$, we briefly comment on the description provided by different classes of DFT functionals of equilibrium structural, elastic, electronic, and dielectric properties. Table I reports such properties as computed with four functionals (SVWN, PBE, B3LYP, and PBE0), belonging to three rungs of "Jacob's ladder," along with their experimental counterparts. Experimental values for lattice parameters $a$ and $c$, and fractional coordinates $(x, y, z)$ of the oxygen atom in general position are taken from the single-crystal X-ray study by Hazen et al. ${ }^{51}$ Voigt's upper bound to the bulk modulus $K_{V}$ is defined in terms of the elastic constants as

$$
K_{V}=\frac{1}{9}\left[2 C_{11}+C_{33}+2\left(C_{12}+2 C_{13}\right)\right] .
$$

The experimental value reported in the table $(89.4 \mathrm{GPa})$ refers to $0 \mathrm{~K}$ and has been derived from the extrapolated elastic constants measured in the temperature range $4.2-300 \mathrm{~K}$ by Farley and Saunders; ${ }^{52}$ note that computed values also refer to $0 \mathrm{~K}$ but neglect zero-point motion effects which, on the contrary, are included in the experimental counterpart. Ordinary $n_{o}$ and extraordinary $n_{e}$ refractive indices, and the corresponding birefringence $\delta=n_{e}-n_{o}$ are taken from Bond ${ }^{53}$ and from Houston et al. ${ }^{54}$ and correspond to an electric field wavelength $\lambda=2500 \mathrm{~nm}$, while theoretical values refer to the $\lambda \rightarrow \infty$ limit (see below for a theoretical investigation of the dispersion of refractive indices with $\lambda$ ).

From inspection of Table I, it is seen that, as expected, the simple LDA functional significantly underestimates the cell size ( $a$ by $1.6 \%$ and $c$ by $1.4 \%$ ), overestimates the bulk modulus (by almost $20 \mathrm{GPa}$, corresponding to about 22\%), describes a relatively narrow electronic band gap of $3.6 \mathrm{eV}$ (here a reliable experimental reference is not available), and largely overestimates the birefringence $\delta$ (by more than a factor of 2). The generalized-gradient PBE functional provides a slightly better description of the lattice parameters, underestimating $a$ by $0.4 \%$ and overestimating $c$ by $2 \%$, a similar description of the electronic band-gap $(3.7 \mathrm{eV})$ but significantly improves upon LDA in the description of the bulk modulus (with an underestimation of about $4.5 \%$ ) and of the birefringence. Hybrid functionals provide the best overall description of all considered properties, with PBE0 slightly outperforming B3LYP. Indeed, PBE0 gives an excellent description of the $a$ lattice parameter (with a deviation

TABLE I. Lattice parameters $a$ and $c$ (in $\AA$ ), fractional coordinates $(x, y, z)$ of the oxygen atom in general position, Voigt bulk modulus $K_{V}$ (in $\mathrm{GPa}$ ), electronic band gap $E_{g}$ (in $\mathrm{eV}$ ), ordinary $n_{o}$ and extraordinary $n_{e}$ refractive indices, and birefringence $\delta$ of $\mathrm{CaWO}_{4}$. Computed values, as obtained with four functionals, are compared with experimental data from Refs. 51-54.

\begin{tabular}{lcccccccccc}
\hline \hline & $a$ & $c$ & $x$ & $y$ & $z$ & $K_{V}$ & $E_{g}$ & $n_{o}$ & $n_{e}$ & $\delta$ \\
\hline SVWN & 5.158 & 11.210 & 0.237 & 0.093 & 0.038 & 108.6 & 3.6 & 1.878 & 1.908 & 0.030 \\
PBE & 5.221 & 11.660 & 0.239 & 0.103 & 0.041 & 85.2 & 3.7 & 1.806 & 1.823 & 0.017 \\
B3LYP & 5.277 & 11.649 & 0.236 & 0.105 & 0.042 & 86.1 & 5.8 & 1.725 & 1.732 & 0.007 \\
PBE0 & 5.241 & 11.516 & 0.236 & 0.102 & 0.041 & 90.4 & 6.2 & 1.736 & 1.747 & 0.011 \\
Expt. & 5.243 & 11.374 & 0.241 & 0.099 & 0.039 & 89.4 & $\ldots$ & 1.872 & 1.885 & 0.013 \\
\hline \hline
\end{tabular}


smaller than $0.04 \%$ ), only slightly overestimating the $c$ one (by $1.2 \%$ ), reliably describes the optical anisotropy of the system (with a $\delta$ of 0.011 with respect to the experimental value of 0.013 ) and provides an excellent description of the elasticity of the system, with a bulk modulus of $90.4 \mathrm{GPa}$ with respect to the experimental value of $89.4 \mathrm{GPa}$; an overestimation by about $1 \%$ is perfectly compatible with the reduction of the bulk modulus that would be induced by the neglected zeropoint motion and that could be evaluated by quasi-harmonic calculations. ${ }^{55-59}$ As expected, hybrid functionals describe a significantly larger gap $E_{g}(5.8-6.2 \mathrm{eV})$ than local-density and generalized-gradient approximations. All functional give a good description of the atomic positions within the cell.

\section{A. The elastic response}

As recalled in Section II A, a prerequisite to a reliable description of the piezo-optics of a crystal is a good calculation of its anisotropic elastic response. In Table II, we report the seven symmetry-independent elastic stiffness $C_{v u}$ and compliance $S_{v u}$ constants of tetragonal $\mathrm{CaWO}_{4}$. The shape of the full fourth-rank elastic tensor for this system is given in Equation (5). In the table, the elastic constants are reported as computed with four DFT functionals and compared with two experimental determinations (one at room temperature and one extrapolated down to $0 \mathrm{~K}$ from data collected in the $4.2-300 \mathrm{~K}$ temperature range).$^{52}$ As expected from the overall structural description given in Table I, the LDA functional is seen to systematically overestimate the $C_{v u}$ and underestimate the $S_{v u}$ constants. The PBE generalized-gradient functional tends to slightly underestimate the elastic stiffness constants $C_{v u}$ while hybrid functionals (more so PBE0 than B3LYP) do provide a satisfactory description of low-temperature elastic constants. As the experimental piezo-optic constants were measured at room temperature, it is interesting to illustrate the effect of temperature on the measured elastic constants. From inspection of the table, we can see that passing from $0 \mathrm{~K}$ to $300 \mathrm{~K}$ the absolute values of all $C_{v u}$ constants are systematically reduced by about $4 \%$ (a converse increase of about $4 \%$ of $S_{v u}$ constants is observed).

A better insight into the description provided by different DFT functionals of the anisotropy of the elastic response of a crystal can be achieved by analyzing directional elastic properties, such as directional seismic wave velocities (i.e., the velocities with which elastic waves do propagate within a crystal along different crystallographic directions). From a fundamental point of view, within the elastic continuum model, the velocity of propagation of a seismic wave traveling along any general crystallographic direction represented by unit wave-vector $\hat{\mathbf{q}}$, can be obtained from the elastic tensor via Christoffel's equation, which can be given an eigenvalues/ eigenvectors form as follows: ${ }^{8,60}$

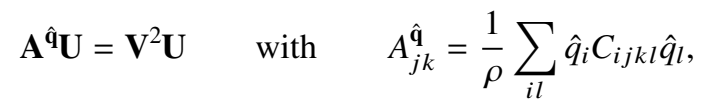

where $A_{j k}^{\hat{\mathrm{q}}}$ is Christoffel's matrix, $\rho$ the crystal density, $i, j, k, l$ $=x, y, z$ represent Cartesian directions, $\hat{q}_{i}$ is the $i$ th element of the unit vector $\hat{\mathbf{q}}, \mathbf{V}$ is a $3 \times 3$ diagonal matrix whose three elements give the acoustic velocities, and $\mathbf{U}=\left(\hat{\mathbf{u}}_{1}, \hat{\mathbf{u}}_{2}, \hat{\mathbf{u}}_{3}\right)$ is the eigenvector $3 \times 3$ matrix where each column represents the polarization $\hat{\mathbf{u}}$ of the corresponding eigenvalue. The three acoustic wave velocities, also referred to as seismic velocities, can be labeled as quasi-longitudinal $v_{p}$, slow quasi-transverse $v_{s 1}$, and fast quasi-transverse $v_{s 2}$, depending on the polarization direction $\hat{\mathbf{u}}$ with respect to wave-vector $\hat{\mathbf{q}}{ }^{61}{ }^{61}$ From directional seismic wave velocities, fundamental aspects of the elastic anisotropy of a crystal, such as shear-wave birefringence and azimuthal anisotropy, can be fruitfully discussed. These data are reported in Figure 1 as computed with the four DFT functionals. We observe that: (i) the four functionals provide a similar overall description of the directional elastic properties of $\mathrm{CaWO}_{4}$; (ii) the crystallographic direction of maximum longitudinal propagation velocity is found between the [110] and the [010] ones while the minimum longitudinal velocity is along the [001] direction; (iii) the direction of maximum propagation velocity of transverse waves is found between the [100] and [110] ones while the minimum is between the [110] and [010] ones; (iv) the basal $a b$ plane is found to be more anisotropic than the vertical ac plane; (v) LDA provides the largest anisotropy, B3LYP the smallest while PBE and PBE0 give a similar and intermediate description of the elastic anisotropy.

\section{B. Photo-elastic and piezo-optic responses}

We can now compare theoretically calculated with experimentally determined stress-optical $\pi_{v u}$ and strain-optical $p_{v u}$ constants of calcium tungstate. However, before doing so, it is worth illustrating how these two sets of data are obtained

- theoretically, strain-optical (photo-elastic) constants are determined as numerical derivatives of the inverse

TABLE II. Symmetry-independent elastic stiffness $C_{v u}$ and compliance $S_{v u}$ constants of tetragonal $\mathrm{CaWO}_{4}$, as computed with four different DFT functionals and compared to experimental data from Farley and Saunders ${ }^{52}$ as measured at $300 \mathrm{~K}$ and extrapolated to $0 \mathrm{~K}$.

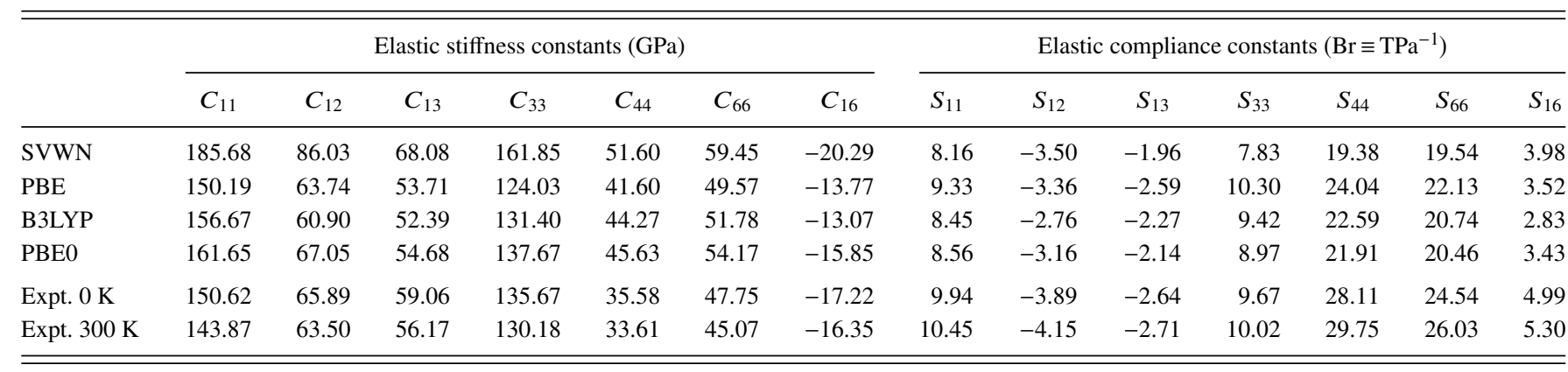




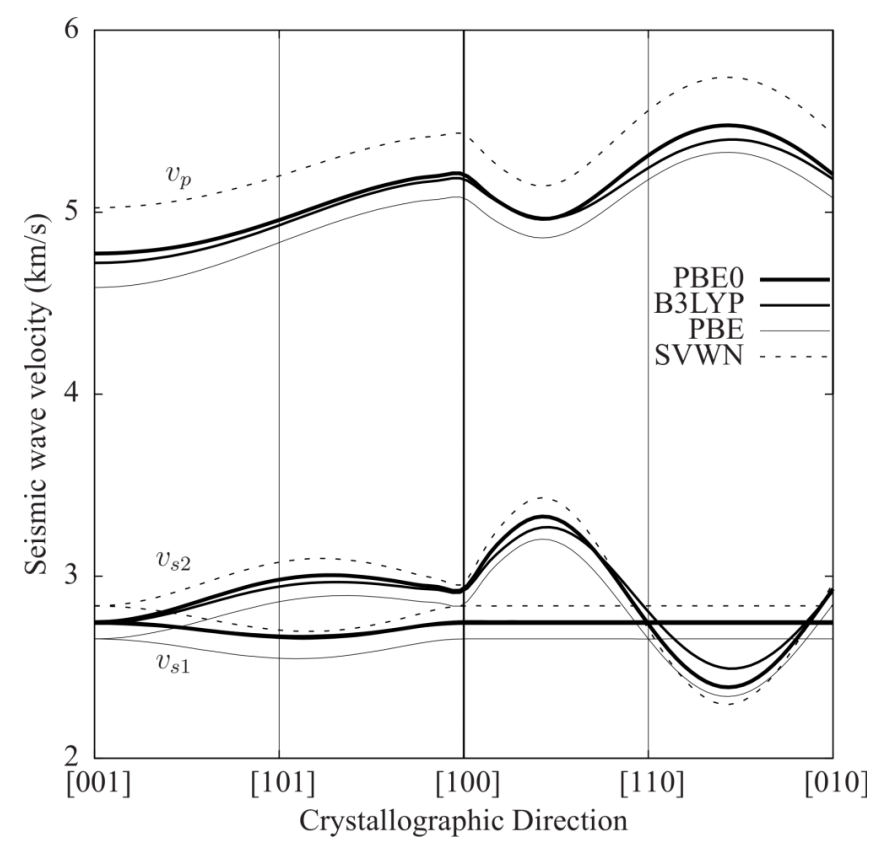

FIG. 1. Directional seismic wave velocities of tetragonal $\mathrm{CaWO}_{4}$ as computed with four different DFT functionals.

of the dielectric tensor with respect to an applied strain according to Equation (7). Stress-optical constants are then obtained through the computed elastic compliance constants $(\boldsymbol{\pi}=\mathbf{p} \mathbb{S})$;

- experimentally, mechanical stresses are measured on five differently cut samples, ${ }^{18}$ previously experimentally determined elastic compliances ${ }^{52} S_{v u}$ and refractive indices ${ }^{62} n_{o}$ and $n_{e}$ are then used to solve a set of equations that provide stress-optical $\pi_{v u}$ constants. Strain-optical constants are determined by combining these stress-optical constants with the elastic stiffness ones $C_{v u}$ previously experimentally measured $(\mathbf{p}=\boldsymbol{\pi} \mathbb{C})$.

Let us stress that if on the one hand the theoretical determination of $p_{v u}$ and $\pi_{v u}$ constants is achieved with a single calculation within a homogeneous computational setup, on the other hand experimental determinations of such properties require a rather complex combination of measurements of different quantities (mechanical stresses, elastic constants, refractive indices) on different samples, which are generally performed in different studies, by different groups, in different conditions, and, above all, with different accuracies. Bearing these considerations in mind, in Table III we report the ten symmetry-independent strain- and stress-optical constants (see Equation (11) for the shape of the fourth-rank $\mathbf{p}$ and $\boldsymbol{\pi}$ tensors) as computed with the four DFT functionals and as compared with available experimental determinations. The full set of piezo-optic $\pi_{v u}$ constants has recently been reported by Mytsyk et al. ${ }^{18}$ where just the sign of the $\pi_{61}$ constant could not be determined while its absolute value was reported to be $\pi_{61}=|0.16|$ Br. Demyanyshyn et al. ${ }^{13}$ have then coupled such constants to the elastic stiffness ones to determine the photo-elastic $p_{v u}$ constants: due to the uncertainty on the sign of the $\pi_{61}$ constant, two photo-elastic constants, $p_{61}$ and $p_{66}$, were not uniquely determined (for $\pi_{61}=-0.16 \mathrm{Br}$ they got $p_{61}=-0.001$ and $p_{66}=-0.018$, while for $\pi_{61}=+0.16 \mathrm{Br}$ they obtained $p_{61}$ $=0.025$ and $\left.p_{66}=-0.031\right)$.

Regarding the effect of DFT functional on such computed properties, from Table III we see that: (i) the computed values of $p_{v u}$ constants obtained with all functionals are rather consistent with each other, particularly so if generalized-gradient (PBE) and hybrid (B3LYP and PBE0) functionals are considered (LDA providing slightly deviating results for some constants, such as $p_{12}, p_{13}, p_{16}$, and $p_{66}$ ); (ii) a similar trend is also observed for $\pi_{v u}$ constants where LDA deviates more from the other functionals due to its poorer description of the elastic

TABLE III. Photoelastic $p_{v u}$ and piezo-optic $\pi_{v u}$ symmetry-independent constants of $\mathrm{CaWO}_{4}$ as computed with four different DFT functionals and as experimentally determined by Mytsyk and co-workers (see text for details). ${ }^{13,17-19}$ Depending on the sign of the $\pi_{61}$ constant (experimentally ill-determined), different experimental values of $p_{61}$ and $p_{66}$ are found (values in parentheses correspond to $\pi_{61}=-0.16 \mathrm{Br}$ while the others to $\left.\pi_{61}=0.16 \mathrm{Br}\right)$.

\begin{tabular}{|c|c|c|c|c|c|c|c|c|c|c|}
\hline & \multicolumn{10}{|c|}{ Photoelastic constants } \\
\hline & $p_{11}$ & $p_{12}$ & $p_{13}$ & $p_{31}$ & $p_{33}$ & $p_{44}$ & $p_{45}$ & $p_{16}$ & $p_{61}$ & $p_{66}$ \\
\hline SVWN & 0.154 & 0.150 & 0.263 & 0.254 & 0.201 & 0.018 & -0.018 & 0.051 & 0.029 & -0.071 \\
\hline PBE & 0.157 & 0.175 & 0.249 & 0.256 & 0.226 & 0.018 & -0.017 & 0.022 & 0.026 & -0.058 \\
\hline B3LYP & 0.152 & 0.194 & 0.240 & 0.264 & 0.208 & 0.009 & -0.017 & 0.019 & 0.026 & -0.050 \\
\hline PBE0 & 0.177 & 0.185 & 0.255 & 0.265 & 0.229 & 0.014 & -0.019 & 0.032 & 0.025 & -0.055 \\
\hline \multirow[t]{4}{*}{ Expt. } & 0.40 & -0.02 & 0.24 & 0.25 & 0.21 & 0.011 & -0.06 & -0.27 & 0.025 & -0.031 \\
\hline & & & & & & & & & $(-0.001)$ & $(-0.018)$ \\
\hline & \multicolumn{10}{|c|}{ Piezo-optic constants $\left(\mathrm{Br} \equiv \mathrm{TPa}^{-1}\right)$} \\
\hline & $\pi_{11}$ & $\pi_{12}$ & $\pi_{13}$ & $\pi_{31}$ & $\pi_{33}$ & $\pi_{44}$ & $\pi_{45}$ & $\pi_{16}$ & $\pi_{61}$ & $\pi_{66}$ \\
\hline SVWN & 0.417 & -0.035 & 1.467 & 0.788 & 0.578 & 0.352 & -0.345 & 1.006 & 0.058 & -1.151 \\
\hline PBE & 0.308 & 0.387 & 1.706 & 0.946 & 1.001 & 0.436 & -0.404 & 0.430 & 0.128 & -1.104 \\
\hline B3LYP & 0.256 & 0.622 & 1.480 & 1.030 & 0.759 & 0.202 & -0.384 & 0.283 & 0.149 & -0.896 \\
\hline PBE0 & 0.495 & 0.367 & 1.508 & 0.938 & 0.922 & 0.318 & -0.409 & 0.637 & 0.100 & -0.964 \\
\hline Expt. & 1.86 & -0.60 & 1.52 & 1.02 & 1.01 & 0.33 & -1.77 & -5.64 & $|0.16|$ & -0.63 \\
\hline
\end{tabular}


compliance constants; furthermore, given the different description of elastic constants provided by PBE, B3LYP, and PBE0, larger differences (never exceeding a factor of 2.2 in the worst cases, though) are found on predicted piezo-optic constants by these functionals; (iii) given the overall better description of elastic constants (see Table II) and refractive indices (see Table I), hybrid functionals are expected to constitute the best choice for the calculation of these properties and to provide the most reliable results.

In order to compare computed constants with experimentally derived ones, let us group the 10 symmetry-independent components of both the photo-elastic and piezo-optic tensors into two sub-sets: a first sub-set contains the $x_{13}, x_{31}, x_{33}, x_{44}$, $x_{61}$, and $x_{66}$ constants while the second sub-set contains the $x_{11}, x_{12}, x_{45}$, and $x_{16}$ ones, where $x$ can be either $p$ or $\pi$. For the first sub-set, which contains 6 constants, considering how delicate both the calculation and the experimental determination of these constants are, the agreement between computed and experimental values is very satisfactory, particularly so if hybrid functionals are considered, as expected. Such a good agreement is a strong evidence of the accuracy of both the experimental reconstruction of these constants and the automated computational strategy here devised. Present calculations allow for the ambiguous determination of the sign of the $\pi_{61}$ constant, which is indeed found to be positive by all functionals. Furthermore we find that by taking the positive sign of this constant, the experimental values of the $p_{61}$ $(0.025)$ and $p_{66}(-0.031)$ constants are also found to be in much better agreement with present calculations. The small residual deviations between computed and experimental constants for this set might be due to either the neglect of thermal effects on computed elastic properties (which, however, is expected to be rather small: about $4 \%$ as noticed above) or the finite value of the electric-field wavelength used in the experiments versus the $\lambda=\infty$ of the calculations (see Section III C for an explicit investigation of such effect).

The situation is completely different when the second subset of 4 constants is considered. In this case, indeed, there is little agreement between computed and experimentally derived values, with very large differences on both absolute values and signs. Given that all computed constants are obtained with the same numerical accuracy, we have to deduce that the experimental reconstruction of these four constants is not as reliable as it was for the other six. On the contrary, present calculations do provide a complete, homogeneous set of all of the ten symmetry-independent photo-elastic and piezo-optic constants of $\mathrm{CaWO}_{4}$.

\section{Effect of the electric field wavelength}

As mentioned at the end of Section III B, experimental measurements of optical properties of crystals are performed using a finite value of the wavelength $\lambda$ of the electric field, whereas theoretical calculations generally refer to the $\lambda=\infty$ case. For instance, all of the experimental photo-elastic and piezo-optic constants of calcium tungstate reported in Table III correspond to $\lambda=633 \mathrm{~nm}$. One might wonder if some of the discrepancies between computed and experimentally derived constants could be ascribed to such an issue. Electric field
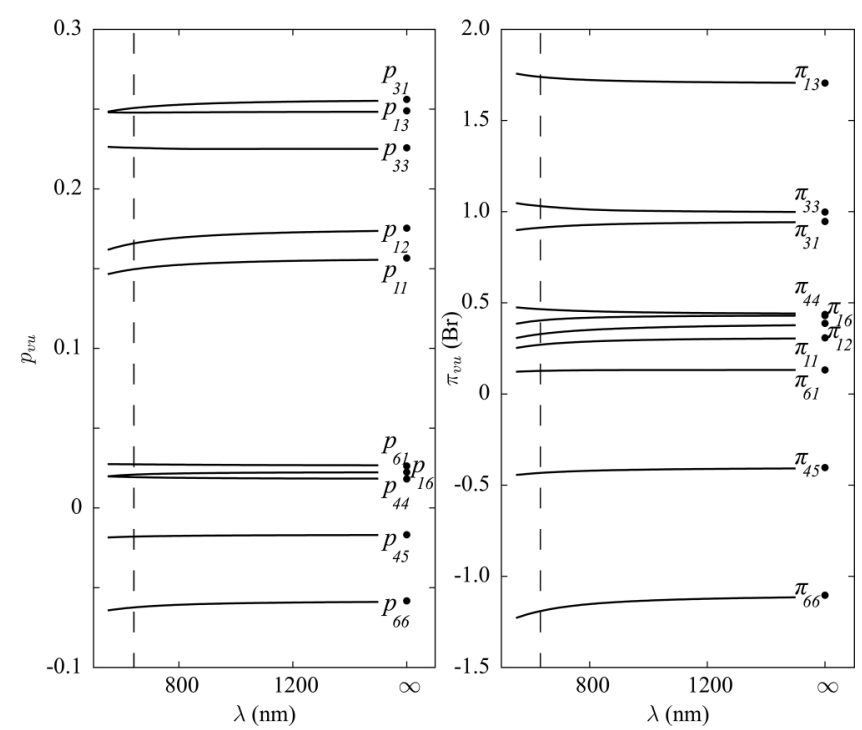

FIG. 2. Dependence on electric field wavelength $\lambda$ of computed photoelastic $p_{v u}$ and piezo-optic $\pi_{v u}$ constants of $\mathrm{CaWO}_{4}$. The dashed vertical lines mark the experimental wavelength $(\lambda=633 \mathrm{~nm})$ used in the determination of the constants reported in Table III. Values obtained with the PBE functional. Computed values for $\lambda=\infty$ are represented by full circles.

frequency dependent optical properties of crystals can be computed with a "dynamical" formulation of the CPHF/KS approach. ${ }^{31,43,44}$ The present algorithm for the calculation of photo-elastic and piezo-optic tensors has been generalized to such a case too. In Figure 2, we report computed $p_{v u}$ and $\pi_{v u}$ constants of $\mathrm{CaWO}_{4}$ (just at the PBE level as similar trends are observed also for other functionals) as a function of $\lambda$ in the range 500-1500 $\mathrm{nm}$ (continuous lines) while static values obtained at $\lambda=\infty$ are given as full circles. A dashed vertical line marks the $\lambda=633 \mathrm{~nm}$ value. We can clearly see that the dispersion of all computed stress-optical and strain-optical constants with $\lambda$ is relatively small (i.e., their values change by just few percents passing from $\lambda=\infty$ to $633 \mathrm{~nm}$ ). As regards photo-elastic constants, $p_{11}$ and $p_{12}$ show the largest dispersion, followed by $p_{31}$ and $p_{66}$ while the other constants are almost flat. Piezo-optic constants show a slightly larger dispersion, $\pi_{66}$ exhibiting the largest among them. Overall, given the small variations of all constants, the electric field frequency dispersion can be ruled out as a possible reason for the large inconsistency of computed and experimental constants of the second sub-set discussed in Section III B.

\section{CONCLUSIONS}

A computational strategy has been devised and implemented in a fully automated fashion for the quantum-mechanical prediction of piezo-optic fourth-rank tensors of crystals belonging to any space group of symmetry. This strategy is based on the simultaneous determination of elastic and photoelastic tensors. A Coupled-Perturbed-Hartree-Fock/KohnSham approach is used to compute the dielectric tensor of strained and unstrained configurations both in the static (infinite electric field wavelength) and dynamic (finite wavelength) cases. Both point and translation symmetries are fully exploited thus drastically reducing the number of independent 
components to be determined and the computational cost of all steps of the calculation.

The scheme has been applied to the $\mathrm{CaWO}_{4}$ calcium tungstate crystal and the full set of 10 symmetry-independent photo-elastic and piezo-optic constants has been determined using four different formulations of the exchange-correlation functional within the density-functional-theory. Present results validate a sub-set of 6 experimentally reconstructed symmetryindependent constants $\left(x_{13}, x_{31}, x_{33}, x_{44}, x_{61}\right.$, and $x_{66}$, being $x$ either the photo-elastic tensor $p$ or the piezo-optic tensor $\pi$ ) and provide more reliable values for a second sub-set of 4 constants $\left(x_{11}, x_{12}, x_{45}\right.$, and $\left.x_{16}\right)$.

${ }^{1}$ J. F. Nye, Physical Properties of Crystals (Oxford University Press, Oxford, 1957).

${ }^{2}$ H.-J. Weber, Phys. Rev. B 51, 12209 (1995).

${ }^{3}$ O. Krupych, V. Savaryn, A. Krupych, I. Klymiv, and R. Vlokh, Appl. Opt. 52, 4054 (2013).

${ }^{4}$ T. S. Narasimhamurty, Photoelastic and Electrooptic Properties of Crystals (Plenum, 1981).

${ }^{5}$ I. Slezinger, A. Alievskaya, and Y. Mironov, Meas. Tech. 28, 1059 (1985).

${ }^{6}$ J. Badoz, M. Billardon, J. C. Canit, and M. F. Russel, J. Opt. 8, 373 (1977).

${ }^{7}$ J. C. Kemp, J. Opt. Soc. Am. 59, 950 (1969).

${ }^{8}$ B. A. Auld, Acoustic Fields and Waves in Solids (Krieger Publishing Company, Malabar, Florida, 1973).

${ }^{9} \mathrm{~J}$. Xu and R. Stroud, Acousto-Optic Devices: Principles, Design, and Applications (Wiley, 1992).

${ }^{10}$ A. S. Andrushchak, B. G. Mytsyk, H. P. Laba, O. V. Yurkevych, I. M. Solskii, A. V. Kityk, and B. Sahraoui, J. Appl. Phys. 106, 073510 (2009).

${ }^{11}$ B. G. Mytsyk, A. S. Andrushchak, N. M. Demyanyshyn, Y. P. Kost', A. V. Kityk, P. Mandracci, and W. Schranz, Appl. Opt. 48, 1904 (2009).

${ }^{12}$ A. Andrushchak, Y. Bobitski, M. Kaidan, B. Mytsyk, A. Kityk, and W. Schranz, Opt. Laser Technol. 37, 319 (2005).

${ }^{13}$ N. M. Demyanyshyn, B. G. Mytsyk, Y. P. Kost, I. M. Solskii, and O. M. Sakharuk, Appl. Opt. 54, 2347 (2015).

${ }^{14}$ H. Z. Cummins and P. E. Schoen, in Laser Handbook, edited by F. T. Arecchi and E. O. Schulz-Dubois (North-Holland Publishing Company, Amsterdam, 1972), p. 1029.

${ }^{15}$ D. Landheer, H. E. Jackson, R. A. McLaren, and B. P. Stoicheff, Phys. Rev. B 13, 888 (1976).

${ }^{16}$ O. Krupych, V. Savaryn, and R. Vlokh, Appl. Opt. 53, B1 (2014).

${ }^{17}$ B. Mytsyk, N. Demyanyshyn, A. Andrushchak, Y. Kost', O. Parasyuk, and A. Kityk, Opt. Mater. 33, 26 (2010).

${ }^{18}$ B. Mytsyk, Ya. P. Kost', N. Demyanyshyn, A. Andrushchak, and I. Solskii, Crystallogr. Rep. 60, 130 (2015).

${ }^{19}$ B. Mytsyk, Y. Kost, N. Demyanyshyn, V. Gaba, and O. Sakharuk, Opt. Mater. 39, 69 (2015)

${ }^{20}$ R. Dovesi, Int. J. Quantum Chem. 29, 1755 (1986).

${ }^{21}$ C. Zicovich-Wilson and R. Dovesi, Int. J. Quantum Chem. 67, 299 (1998).

${ }^{22}$ C. Zicovich-Wilson and R. Dovesi, Int. J. Quantum Chem. 67, 311 (1998).

${ }^{23}$ R. Orlando, M. D. L. Pierre, C. M. Zicovich-Wilson, A. Erba, and R. Dovesi, J. Chem. Phys. 141, 104108 (2014).

${ }^{24}$ K. Doll, N. M. Harrison, and V. R. Saunders, Int. J. Quantum Chem. 82, 1 (2001).

${ }^{25}$ K. Doll, Comput. Phys. Commun. 137, 74 (2001).

${ }^{26}$ K. Doll, R. Dovesi, and R. Orlando, Theor. Chem. Acc. 112, 394 (2004).

${ }^{27}$ K. Doll, R. Dovesi, and R. Orlando, Theor. Chem. Acc. 115, 354 (2006).
${ }^{28}$ M. Ferrero, M. Rérat, R. Orlando, and R. Dovesi, J. Comput. Chem. 29, 1450 (2008).

${ }^{29}$ M. Ferrero, M. Rérat, R. Orlando, and R. Dovesi, J. Chem. Phys. 128, 014110 (2008).

${ }^{30}$ M. Ferrero, M. Rérat, B. Kirtman, and R. Dovesi, J. Chem. Phys. 129, 244110 (2008).

${ }^{31}$ R. Dovesi, R. Orlando, A. Erba, C. M. Zicovich-Wilson, B. Civalleri, S. Casassa, L. Maschio, M. Ferrabone, M. De La Pierre, Ph. D'Arco et al., Int. J. Quantum Chem. 114, 1287 (2014).

${ }^{32}$ R. Dovesi, V. R. Saunders, C. Roetti, R. Orlando, C. M. Zicovich-Wilson, F. Pascale, K. Doll, N. M. Harrison, B. Civalleri, I. J. Bush et al., CRYSTAL14 User's Manual, Università di Torino, Torino, 2014, http://www.crystal. unito.it.

${ }^{33}$ A. Erba, A. Mahmoud, R. Orlando, and R. Dovesi, Phys. Chem. Miner. 41, 151 (2014).

${ }^{34}$ A. Erba, A. Mahmoud, D. Belmonte, and R. Dovesi, J. Chem. Phys. 140, 124703 (2014)

${ }^{35}$ A. Erba, K. E. El-Kelany, M. Ferrero, I. Baraille, and M. Rérat, Phys. Rev. B 88, 035102 (2013).

${ }^{36}$ K. E. El-Kelany, P. Carbonnière, A. Erba, and M. Rérat, J. Phys. Chem. C 119, 8966 (2015).

${ }^{37}$ A. Erba, M. Ferrabone, J. Baima, R. Orlando, M. Rérat, and R. Dovesi, J. Chem. Phys. 138, 054906 (2013).

${ }^{38}$ A. Erba and R. Dovesi, Phys. Rev. B 88, 045121 (2013).

${ }^{39}$ J. P. Perdew and K. Schmidt, AIP Conf. Proc. 577, 1 (2001).

${ }^{40}$ W. F. Perger, J. Criswell, B. Civalleri, and R. Dovesi, Comput. Phys. Commun. 180, 1753 (2009).

${ }^{41}$ G. Saghi-Szabo, R. E. Cohen, and H. Krakauer, Phys. Rev. Lett. 80, 4321 (1998).

${ }^{42}$ A. Dal Corso, M. Posternak, R. Resta, and A. Baldereschi, Phys. Rev. B 50, 10715 (1994).

${ }^{43}$ L. Bernasconi, S. Tomić, M. Ferrero, M. Rérat, R. Orlando, R. Dovesi, and N. M. Harrison, Phys. Rev. B 83, 195325 (2011).

${ }^{44}$ A. Ferrari, R. Orlando, and M. Rérat, J. Chem. Theor. Comput. 11(7), 3245-3258 (2015)

${ }^{45}$ F. Corá, A. Patel, N. M. Harrison, R. Dovesi, and C. R. A. Catlow, J. Am. Chem. Soc. 118, 12174 (1996).

${ }^{46}$ J. C. Slater, Phys. Rev. 81, 385 (1951).

${ }^{47}$ S. H. Vosko, L. Wilk, and M. Nusair, Can. J. Phys. 58, 1200 (1980).

${ }^{48}$ J. P. Perdew, K. Burke, and M. Ernzerhof, Phys. Rev. Lett. 77, 3865 (1996).

${ }^{49}$ A. D. Becke, J. Chem. Phys. 98, 5648 (1993).

${ }^{50} \mathrm{C}$. Adamo and V. Barone, J. Chem. Phys. 110, 6158 (1999).

${ }^{51}$ R. M. Hazen, L. W. Finger, and J. W. Mariathasan, J. Phys. Chem. Solids 46, 253 (1985).

${ }^{52}$ J. Farley and G. Saunders, Solid State Commun. 9, 965 (1971).

${ }^{53}$ W. L. Bond, J. Appl. Phys. 36, 1674 (1965).

${ }^{54}$ T. W. Houston, L. F. Johnson, P. Kisliuk, and D. J. Walsh, J. Opt. Soc. Am. 53, 1286 (1963).

${ }_{55}^{5}$ A. Erba, J. Chem. Phys. 141, 124115 (2014).

${ }^{56}$ A. Erba, M. Shahrokhi, R. Moradian, and R. Dovesi, J. Chem. Phys. 142, 044114 (2015).

${ }^{57}$ A. Erba, J. Maul, R. Demichelis, and R. Dovesi, Phys. Chem. Chem. Phys. 17, 11670 (2015).

${ }^{58}$ A. Erba, J. Maul, M. De La Pierre, and R. Dovesi, J. Chem. Phys. 142, 204502 (2015).

${ }^{59}$ A. Erba, J. Maul, M. Itou, R. Dovesi, and Y. Sakurai, Phys. Rev. Lett. 115, 117402 (2015).

${ }^{60}$ M. J. P. Musgrave, Crystal Acoustics (Holden-Day, San Francisco, California, 1970).

${ }^{61}$ B. B. Karki, L. Stixrude, and R. M. Wentzcovitch, Rev. Geophys. 39, 507, doi:10.1029/2000RG000088 (2001)

${ }^{62}$ T. A. Davis and K. Vedam, J. Opt. Soc. Am. 58, 1446 (1968). 